\title{
Some Applications of Order Statistics in Inventory Control
}

\author{
Dr.T.Venkatesan ${ }^{1}$, Dr.G.Arivazhagan ${ }^{2}$, Dr.C.Muthu ${ }^{3}$ \\ ${ }^{I}$ (Assistant Professor, Department of Statistics,St.Joseph's College, Trichy, Tamil Nadu / India) \\ ${ }_{2}^{2}$ (Assistant Professor, Department of Statistics, Raja Serfij Govt College (Autonomous), Thanjavur, Tamil Nadu) \\ 3(Associate Professor, Department of Statistics,St.Joseph's College,Trichy, Tamil Nadu / India)
}

\begin{abstract}
Among the various inventory systems, the stations in series is an interesting one. There may be a number of nodes in between the starting point or node and the end point or final node. The production process starts at the first node and the finished products are at the last node. The output of the previous node happens to be the input for the next. Using this concept a model with two machines $M_{1}$ and $M_{2}$ in series are considered and there is an inventory of semi finished products in order to keep uninterrupted supply to the machine at the end point. This is due to possibility of breakdown of the machine at the first node. In this paper the optimal reserve inventory between the two machines is obtained assuming that the breakdown duration of $M_{1}$ is a random variable follows the first order statistic. Similarly, this problem can be extended to the case of three machines in series and in such a case the reserve $S_{1}$ is the quantity of semi finished product generated by $M_{1}$ and $S_{2}$ stands for the quantity of semi finished product by $M_{2}$. The optimal size of the reserves $S_{1}$ and $S_{2}$ are obtained under the assumption that the breakdown duration of the first machine $M_{1}$ is a random variable distributed as first order statistic.
\end{abstract}

Keywords: Optimal reserve, Semi finished products, Machines in series, First order statistic.

\section{Optimal Reserve inventory between two machines in series}

There are many manufacturing industries in which the methods of inventory control need to be applied. The optimal stock size of the raw materials, the semi finished products and also the finished products are a matter of vital importance. The profits earned by the industry depend upon the use of appropriate stock management which requires the appropriate application of inventory policies. In this paper a model for the determination of optimal reserve inventory between two machines in series is discussed.

A system in which there are two machines $M_{1}$ and $M_{2}$ in series is considered. The output of $M_{1}$ is the input for the following is the configuration of the system.

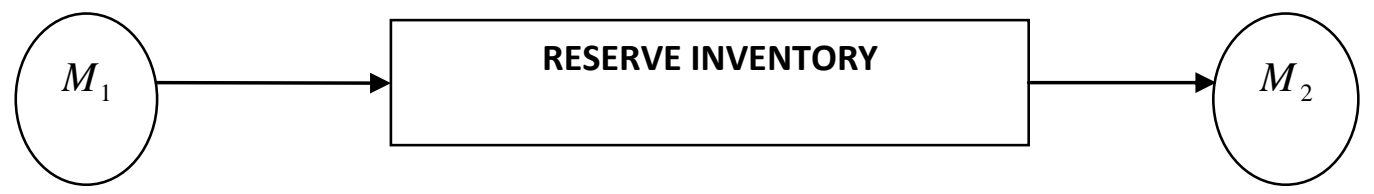

Whenever the machine $M_{1}$ suffers a breakdown, then the supply of raw materials to $M_{2}$ is not available and is forced to be in down or idle state. The consumption rate of $M_{2}$ is a constant ' $r$ '. The idle time cost of $M_{2}$ is very high and prohibitive. The breakdown duration or repair time of $M_{1}$ is a random variable. To avoid the idle time of $M_{2}$ due to the break downs of $M_{1}$ a reserve inventory of output of $M_{1}$ is kept in between $M_{1}$ and $M_{2}$. If on the other hand there is shortage due to inadequate inventory size then $M_{2}$ is forced to go to idle state and it is very costly. The interarrival times between $M_{2}$ the successive break downs of $M_{1}$ are also random variables. The problem is to determine the optimal reserve inventory between $M_{1}$ and $M_{2}$ . The basic model using this concept has been discussed in Hanssmaan (1962) as under:

\subsection{Assumptions}

1. There are two machines in series and the output of $M_{1}$ is the input for $M_{2}$

2. The break downs of $M_{1}$ results in the idle time of $M_{2}$

3. The idle time of $M_{2}$ is costly. 
4. The repair times of machine $M_{1}$ are i.i.d random variables.

5. The inter arrival times between the successive break downs of $M_{1}$ are also i.i.d random variables.

\subsection{Notations}

$\mathrm{h}=$ inventory holding cost per unit / unit time for the reserve.

$\mathrm{d}=$ idle time cost per unit time for $M_{2}$

$\tau=$ a continuous random variable denoting the repair time or break down duration of $M_{1}$ and the p.d.f is $\mathrm{g}($. and c.d.f $\mathrm{G}($.

$\mathrm{r}=$ constant consumption rate of $M_{2}$

$\mathrm{X}=\mathrm{a}$ continuous random variable denoting the inter arrival times between successive break downs of $M_{1}$ and $X \sim \exp (\mu)$

\section{Results}

If the reserve inventory between two machines is denoted as ' $\mathrm{S}$ ' then $\frac{S}{r}$ is the time taken by $M_{2}$ to the exhaust the reserve size of ' $\mathrm{S}$ ' Idle time of

$$
\begin{aligned}
M_{2}=0 & \text { if } & & \tau \leq \frac{S}{r} \\
=\tau-\frac{S}{r} & \text { if } & \tau & >\frac{S}{r}
\end{aligned}
$$

If $\tau \leq \frac{S}{r}$ then the repair of $M_{1}$ is completed before the reserve inventory is exhausted by $M_{2}$. Hence the idle time is zero. If $\tau>\frac{S}{r}$ then the reserve inventory is exhausted before the machine $M_{1}$ is brought to upstate and hence $\tau-\frac{S}{r}$ is the idle time of $M_{2}$.

The average number of break downs of $M_{1}$ per unit of time is $\frac{1}{\mu}$.

The total expected cost per unit of time is

$$
E(C)=h s+\frac{d}{\mu} \int_{\frac{s}{r}}^{\infty}\left(\tau-\frac{S}{r}\right) g(\tau) d \tau
$$

To determine the optimal S, we take

$$
\frac{d E(C)}{d s}=0
$$

Using the Lebinitz rule the optimal solution is obtained as $G\left(\frac{\hat{S}}{r}\right)=1-\frac{r \mu h}{d}$

It may be observed that in the above solution to the model there is a constraint that $\frac{r \mu h}{d}<1$. Otherwise the solution could not be obtained. However if we consider the model as

$$
E(C)=h r \int_{0}^{\frac{s}{r}}\left(\frac{S}{r}-\tau\right) g(\tau) d \tau+\frac{d}{\mu} \int_{\frac{s}{r}}^{\infty}\left(\tau-\frac{S}{r}\right) g(\tau) d \tau
$$


Using Lebinitz rule, we have $G\left(\frac{\hat{S}}{r}\right)=\frac{d}{r \mu h+d}<1$ fo all $d, \mu h$.

It may be noted that there is no constraint on the values of $\mathrm{r}, \mathrm{d}, \mathrm{h}$, and $\mu$ in this model. Ramachandran and Sathiyamoorthy (1981) have given a modified version of this model. Rajagopal and Sathiyamoorthy (2003) have extended this model to the case of the three machines in series. Srinivasan et.al (2007) have discussed a different versions of this model using the concept of order statistics to represent the inter arrival times of successive break downs of machine $M_{1}$.

\section{Revised model I}

A modification of the model discussed here, using the concept of order statistics .It is assumed that the repair time $\tau$ of machine $M_{1}$ is a random variable and hence a sample of $\mathrm{n}$-observations from the value of $\tau$ are taken. These values are arranged in increasing order magnitude. So that $\tau_{(1)} \leq \tau_{(2)} \leq \ldots \ldots . \leq \tau_{(n)}$ for order statistics.

Let $\tau_{(1)}$ denote the first order or minimum order statistic Now assuming the repair time following first order statistic the optimal reserve inventory between $M_{1}$ and $M_{2}$ is obtained as follows

$$
g\left(\tau_{(1)}\right)=n[1-G(\tau)]^{n-1} g(\tau)
$$

Hence

$$
\begin{aligned}
E(C) & =h r \int_{0}^{\frac{s}{r}}\left(\frac{S}{r}-\tau\right) g_{(1)}(\tau) d \tau+\frac{d}{\mu} \int_{\frac{s}{r}}^{\infty}\left(\tau-\frac{S}{r}\right) g_{(1)}(\tau) d \tau \\
& =h r \int_{0}^{s / r}\left(\frac{S}{r}-\tau\right) n[1-G(\tau)]^{n-1} g(\tau) d \tau+\frac{d}{\mu} \int_{s / r}^{\infty}\left(\tau-\frac{S}{r}\right) n[1-G(\tau)]^{n-1} g(\tau) d \tau
\end{aligned}
$$

If $\tau \sim \exp (\theta)$

$$
\begin{aligned}
& \frac{d E(C)}{d s}=0 \quad \text { gives } \\
& e^{-\frac{\theta h s}{r}}=\frac{h}{\left(\frac{r h \mu+d}{\mu r}\right)} \\
& e^{\frac{\theta n s}{r}}=\frac{\mu r h+d}{\mu r h} \\
& \hat{S}=\frac{r}{\theta n} \log \left(1+\frac{d}{\mu r h}\right)
\end{aligned}
$$

This is the optimal reserve inventory between $M_{1}$ and $M_{2}$. 


\section{Numerical illustration}

The changes in $\hat{S}$ for the changes in the inventory holding cost $\mathbf{h}$ is given in the following table (1) $r=20 \mu=1.5 d=100 \mathrm{n}=20 \mathrm{~h} \theta=1.2$ are all fixed

Table 1

\begin{tabular}{|c|l|}
\hline $\mathrm{h}$ & \multicolumn{1}{|c|}{$\hat{\mathrm{S}}$} \\
\hline 10 & 0.551973 \\
\hline 20 & 0.295755 \\
\hline 30 & 0.202096 \\
\hline 40 & 0.153539 \\
\hline 50 & 0.123791 \\
\hline 60 & 0.103831 \\
\hline 70 & 0.089245 \\
\hline 80 & 0.078305 \\
\hline 90 & 0.06986 \\
\hline 100 & 0.062951 \\
\hline
\end{tabular}

Fig. 1

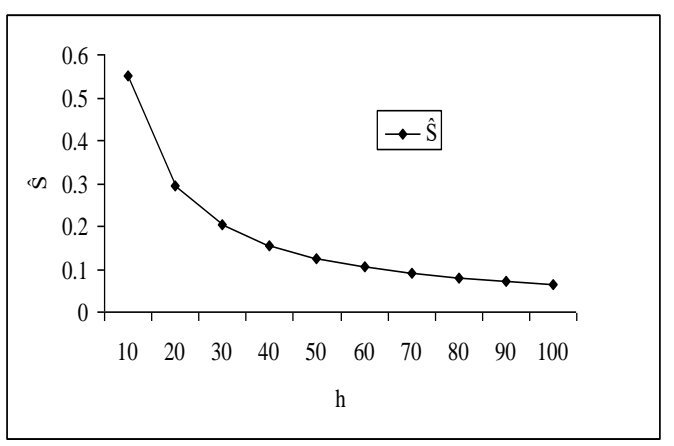

The changes in $\hat{S}$ for the changes in the inventory holding cost d given following table (2) $r=20 \mu=1.5 h=10 n=20 \quad \theta=1.2$ are all fixed

Table -2

Fig-2

\begin{tabular}{|c|r|}
\hline $\mathrm{d}$ & \multicolumn{1}{c|}{$\hat{\mathrm{s}}$} \\
\hline 100 & 0.239713 \\
\hline 120 & 0.280401 \\
\hline 130 & 0.319169 \\
\hline 140 & 0.356211 \\
\hline 150 & 0.391717 \\
\hline 160 & 0.425687 \\
\hline 170 & 0.458506 \\
\hline 180 & 0.48979 \\
\hline 190 & 0.520114 \\
\hline 200 & 0.549478 \\
\hline
\end{tabular}



\section{Determination of optimal reserve of semi finished products between three machines in} series.

It is assumed that there are three machines $M_{1}, M_{2}$ and $M_{3}$ in series. The output of $M_{1}$ is the input for $M_{2}$ and the output of $M_{2}$ is the input for $M_{3}$. The following diagram shows the arrangement.

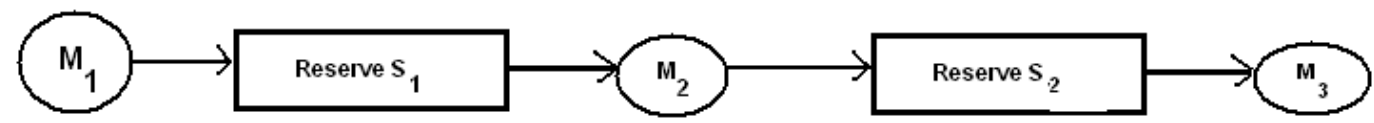

The size of the optimal reserves $S_{1}$ and $S_{2}$ are determined under the assumption that the random duration of breakdown of machine $M_{1}$ is a random variable which follows order statistics 


\subsection{Assumptions:}

1. There are three machines in series . The output of $M_{1}$ is the input for $M_{2}$ and similarly the output of $M_{2}$ is the input for $M_{3}$.

2. Machine $M_{1}$ undergoes breakdown for a random duration. Hence the break down duration or the repair time of $M_{1}$ is a random variable.

3. $M_{2}$ and $M_{3}$ are in upstate, but when the input is not available they are forced to go to the idle state.

4. The inter arrival times between the breakdowns of $M_{1}$ are i.id random variables.

5. The break down duration of $M_{1}$ is taken as a random variable which is distributed as a first order statistics and similarly the $\mathrm{n}^{\text {th }}$ order statistics.

\subsection{Notations}

(i) $r_{1} r_{2}$ denote the consumption rate of $M_{2}, M_{3}$ respectively.

(ii) $h_{1}, h_{2}$ denote the inventory holding costs per unit of $S_{1}$ and $S_{2}$ respectively.

(iii) $d_{1}, d_{2}$ denote the downtime costs of $M_{2}, M_{3}$ respectively.

(iv) $\mu_{1}, \mu_{2}$ denote the average time between breakdowns $M_{1}, M_{2}$ respectively.

(v) $\tau$, denote the down time duration of $M_{1}$, and is a which are random variable that follow first order statistic and similarly $n^{\text {th }}$ order statistic.

(vi) $g_{(1)}(\tau)$ are the p.d.f of first order statistic .

\subsection{Results}

$$
E(C)=h_{1} r_{1} \int_{0}^{s_{1} / r_{1}}\left(\frac{s_{1}}{r_{1}}-\tau\right) g_{(1)}(\tau) d \tau+h_{2} s_{2}+\frac{d_{1}}{\mu_{1}} \int_{s_{1} / r_{1}}^{\infty}\left(\tau-\frac{s_{1}}{r_{1}}\right) g_{(1)}(\tau) d \tau+\frac{d_{2}}{\mu_{1}} \int_{\frac{s_{1}}{r_{1}}}^{\infty} \frac{s_{2}}{r_{r_{2}}}{ }_{s_{2}}\left(\tau-\frac{s_{2}}{r_{2}}-\frac{s_{1}}{r_{1}}\right) g_{(1)}(\tau) d \tau
$$

Since $\tau$ follows $\exp (\lambda)$, the p.d.f of first order statistic is $g_{(1)}(\tau)=n \lambda\left[e^{-\lambda \tau}\right]^{n}$

$$
\begin{aligned}
& E(C)=h_{1} r_{1} \int_{0}^{s_{1} / r_{1}}\left(\frac{s_{1}}{r_{1}}-\tau\right) n \lambda\left[e^{-\lambda \tau}\right]^{n} d \tau+h_{2} s_{2}+\frac{d_{1}}{\mu_{1}} \int_{s_{1} / r_{1}}^{\infty}\left(\tau-\frac{s_{1}}{r_{1}}\right) n \lambda\left[e^{-\lambda \tau}\right]^{n} d \tau+\frac{d_{2}}{\mu_{1}} \int_{\frac{s_{1}}{r_{1}}+\frac{s_{2}}{r_{2}}}^{\infty}\left(\tau-\frac{s_{2}}{r_{2}}-\frac{s_{1}}{r_{1}}\right) n \lambda\left[e^{-\lambda \tau}\right]^{n} d \tau \\
& \frac{d E(C)}{d s_{1}}=\frac{d}{d s_{1}}\left[h_{1} r_{1} n \lambda A+h_{2} s_{2}+\frac{d_{1} n \lambda}{\mu_{1}} B+\frac{d_{2} n \lambda}{\mu_{1}} C\right]=0 \\
& \text { where } \\
& \mathrm{A} \Rightarrow \int_{0}^{s_{1} / r_{1}}\left(\frac{s_{1}}{r_{1}}-\tau\right)\left[e^{-\lambda \tau}\right]^{n} d \tau
\end{aligned}
$$

Using Leibenitz rule for differentiation of an integral, we get

$$
\begin{aligned}
& \mathrm{A}=\frac{1}{n \lambda r_{1}}\left[1-e^{-n \lambda \frac{s_{1}}{r_{1}}}\right] \\
& \mathrm{B} \Rightarrow \int_{s_{1} / r_{1}}^{\infty}\left(\tau-\frac{s_{1}}{r_{1}}\right)\left[e^{-n \lambda \tau}\right] d \tau
\end{aligned}
$$

Similarly 


$$
\begin{aligned}
& \mathrm{B}=\frac{-1}{n \lambda r_{1}}\left[e^{-n \lambda \frac{s_{1}}{r_{1}}}\right] \text { and } \\
& \mathrm{C}=\int_{\frac{s_{1}}{r_{1}}+\frac{s_{2}}{r_{2}}}^{\infty}\left(\tau-\frac{s_{2}}{r_{2}}-\frac{s_{1}}{r_{1}}\right)\left[e^{-\lambda \tau]^{n}} d \tau=0\right. \\
& \mathrm{C}=\frac{-1}{n \lambda r_{1}}\left[e^{-n \lambda\left(\frac{s_{1}}{r_{1}}+\frac{s_{2}}{r_{2}}\right)}\right]
\end{aligned}
$$

Substituting A, B, C in (1)

$$
\begin{aligned}
& h_{1} r_{1} n \lambda \frac{1}{n \lambda r_{1}}\left[1-e^{-n \lambda \frac{s_{1}}{r_{1}}}\right]-\frac{d_{1}}{\mu_{1}} n \lambda \frac{1}{n \lambda r_{1}} e^{-n \lambda \frac{s_{1}}{r_{1}}}-\frac{d_{2}}{\mu_{1}} n \lambda \frac{1}{n \lambda r_{1}} e^{-n \lambda\left(\frac{s_{1}}{r_{1}}+\frac{s_{2}}{r_{2}}\right)}=0 \\
& h_{1}\left[1-e^{-n \lambda \frac{s_{1}}{r_{1}}}\right]-\frac{d_{1}}{\mu_{1} r_{1}} e^{-n \lambda \frac{s_{1}}{r_{1}}}-\frac{d_{2}}{\mu_{1} r_{1}} e^{-n \lambda\left(\frac{s_{1}}{r_{1}}+\frac{s_{2}}{r_{2}}\right)}=0
\end{aligned}
$$

Again

$$
\begin{aligned}
& \frac{d E(C)}{d s_{2}}=0 \Rightarrow \\
& h_{2}+\frac{d_{2}}{\mu_{1}} n \lambda \int_{\frac{s_{1}}{r_{1}}+\frac{s_{2}}{r_{2}}}^{\infty}\left(\tau-\frac{s_{2}}{r_{2}}-\frac{s_{1}}{r_{1}}\right) e^{-n \lambda \tau} d \tau=0 \\
& h_{2}-\frac{d_{2}}{\mu_{1} r_{2}}\left[e^{-n \lambda\left(\frac{s_{1}}{r_{1}}+\frac{s_{2}}{r_{2}}\right)}\right]=0
\end{aligned}
$$

Solving (2) and (3) we get

$$
\begin{aligned}
& e^{-n \lambda \frac{s_{1}}{r_{1}}}=\frac{\mu_{1}\left(h_{1} r_{1}-h_{2} r_{2}\right)}{\left(h_{1} \mu_{1} r_{1}+d_{1}\right)} \\
& \hat{s}_{1}=\frac{r_{1}}{n \lambda} \log \left[\frac{\mu_{1}\left(h_{1} r_{1}-h_{2} r_{2}\right)}{\left(d_{1}+h_{1} \mu_{1} r_{1}\right)}\right]^{-1}
\end{aligned}
$$

on simplification

Again from (3)

$$
\begin{aligned}
& h_{2}-\frac{d_{2}}{\mu_{1} r_{2}}\left[e^{-n \lambda\left(\frac{s_{1}}{r_{1}}+\frac{s_{2}}{r_{2}}\right)}\right]=0 \\
& \hat{s}_{2}=\frac{r_{2}}{n \lambda}\left[\log \left[\frac{h_{2} r_{2}\left(d_{1}+h_{1} \mu_{1} r_{1}\right)}{d_{2}\left(h_{1} r_{1}-h_{2} r_{2}\right)}\right]\right]^{-1}
\end{aligned}
$$




\subsection{Numerical illustration:}

The Changes in $\hat{S}_{1}$ and $\hat{S}_{2}$ for the changes in $\mathrm{h}_{1}$ is given in the following table. $\mathrm{h}_{1}=10, \mathrm{~h}_{2}=5, \mathrm{r}_{1}=20, \mathrm{r}_{2}=5, \mathrm{~d}_{1}=50$, $\mathrm{d}_{2}=100, \lambda=1.5, \mu=1, \mathrm{n}=25$

Table 1

\begin{tabular}{|c|l|l|}
\hline $\mathrm{h}_{1}$ & $\hat{S}_{1}$ & $\hat{S}_{2}$ \\
\hline 10 & 0.082614 & 0.059621 \\
\hline 15 & 0.055858 & 0.066309 \\
\hline 20 & 0.042229 & 0.069717 \\
\hline 25 & 0.033956 & 0.071785 \\
\hline 30 & 0.028397 & 0.073175 \\
\hline 35 & 0.024403 & 0.074173 \\
\hline 40 & 0.021395 & 0.074925 \\
\hline 45 & 0.019048 & 0.075512 \\
\hline 50 & 0.017165 & 0.075983 \\
\hline 55 & 0.015621 & 0.076369 \\
\hline
\end{tabular}

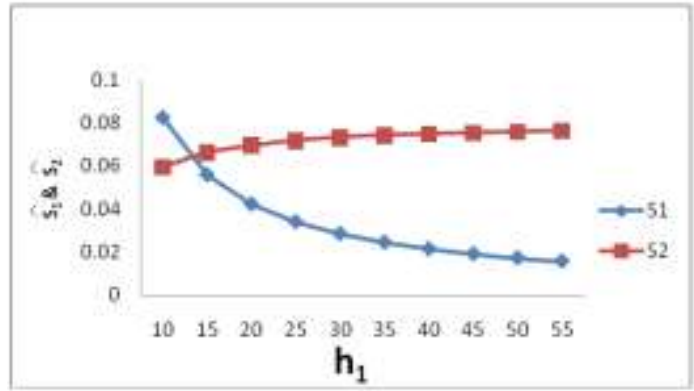

Fig -1

The Changes in $\hat{S}_{1}$ and $\hat{S}_{2}$ for the changes in $\mathrm{h}_{2}$ is given in the following table. $\mathrm{h}_{1}=10, \mathrm{~h}_{2}=5, \mathrm{r}_{1}=20, \mathrm{r}_{2}=5, \mathrm{~d}_{1}=50$, $\mathrm{d}_{2}=100, \lambda=1.5, \mu=1, \mathrm{n}=25$

Table 2

\begin{tabular}{|l|l|l|}
\hline $\mathrm{h}_{2}$ & $\hat{S}_{1}$ & $\hat{S}_{2}$ \\
\hline 5 & 0.082614 & 0.059621 \\
\hline 5.5 & 0.085947 & 0.053268 \\
\hline 6 & 0.089328 & 0.047385 \\
\hline 6.5 & 0.092760 & 0.041892 \\
\hline 7 & 0.096243 & 0.036730 \\
\hline 7.5 & 0.099779 & 0.031850 \\
\hline 8 & 0.103370 & 0.027215 \\
\hline 8.5 & 0.107018 & 0.022793 \\
\hline 9 & 0.110724 & 0.018557 \\
\hline 9.5 & 0.114490 & 0.014484 \\
\hline
\end{tabular}

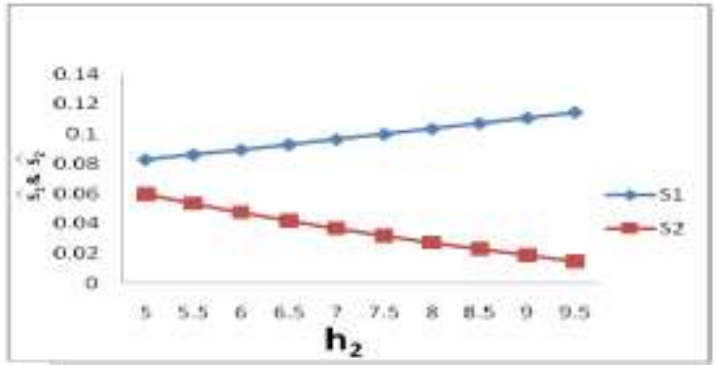

Fig 2

\section{Conclusions for Model I}

1. As the value of ' $\mathrm{h}$ ' namely the inventory holding cost increases there is a decreases in the size of the optimal inventory $\hat{S}$ both in the case of the repair time being first order statistic

2. As the shortage cost ' $d$ ' increases the models suggests that the optimal inventory size namely $\hat{S}$ should be higher. This is found to be true in both the cases namely when the repair time ' $\tau$ ' follows first order statistic

3. It may be noted that as the value of $\theta$ which is the parameter of exponential distribution for the duration of break down ' $\tau$ ' increases it implies that $E(\tau)=\frac{1}{\theta}$ decreases. Therefore the average duration of breakdown is smaller and therefore the repair will be carried out quickly. So a smaller inventory is sufficient so that $\hat{S}$ decreases.

4. When $\mu$ which is the average of the inter arrival times between the breakdowns of $M_{1}$ increases then $\frac{1}{\mu}$ decreases. In other words the average number of breakdowns per unit of time decreases. So a smaller inventory is sufficient. 


\section{Conclusions for Model II}

On the basis of the numerical illustrations worked out the following interesting conclusions are observed. In the case of the breakdown duration being distributed as first order statistic, the following are the conclusions.

1. As the inventory holding cost $h_{1}$ for the reserve at stage 1 namely $S_{1}$ increases, the size of $S_{1}$ decreases which is in agreement with inventory control theory. Also it is seen that if the size of $S_{1}$ is decreasing then a higher level of $S_{2}$ is advocated since the shortage cost due to $M_{3}$ should be minimum.Hence a higher stock level of $S_{2}$ is desirable.Thisis indicated in table (1) and Fig(1).

2. As $h_{2}$ namely the inventory holding cost at stage2 namely that of $S_{2}$ increases then a smaller inventory $S_{2}$ is advocated . but at the same time a higher inventory at stage 1 , namely $S_{1}$ is desirable .

3. If $r_{1}$ which is the consumption rate of $M_{2}$ is on the increase then a small inrease in $S_{1}$ is suggested. Similarly an increase in $S_{2}$ is also indicated. This is due to the fact that if consumption rate is on the increase then a higher reserve will reduce the chance of break down of $M_{2}$ and $M_{3}$.

4. When $r_{2}$ which is the conumption rate of machine $M_{3}$ is on the increase, it is observed that $S_{1}$ increases , but there is a derease in $S_{2}$. This is due to the fact that the overall cost $\mathrm{E}(\mathrm{C})$ should be a minimum.

5. As $d_{1}$ which is the shortage cost associated with $M_{2}$ is on the increase then a higher level of $S_{1}$ is suggested and hence $S_{1}$ increases. But to reduce the overall cost, a decrease in $S_{2}$ is suggested

6. As $d_{2}$ which is the down time or shortage cost of $M_{3}$ increases then a higher level of $S_{2}$ is advocated, but $S_{1}$ is remains the same. Similarly we can Proceed this model for $\mathrm{n}^{\text {th }}$ order statistic

\section{References}

[1]. Hannsmaan (1962). Operations Research in Production and Inventory Control, John Wiley and Sons, Inc. New York.

[2]. Ramachandran and Sathiyamoorthi (1981). Optimal Reserve for two machines, IEEE Trans on Reliability, Vol. R-30, No. 4, p. 397.

[3]. Rajagopal and Sathiyamoorthi (2003). An Extension of the Optimal Reserve Inventory ----- between Machines Model - Proceeding of the Second National Conference on Mathematical and Computational Models - PSG College of Engg., Dec. 11 - 12.

[4]. Srinivasan, Sulaiman and Sathiyamoorthi (2007). Optimal Reserve Inventory Between two machines under SCBZ Property of Interarrival Times between Breakdowns, Ultra Science, Vol. 9 (2) M, pp. $261-266$. 